Check for updates

Clinical Epidemiology Center, Research and Development Service, VA Saint Louis Health Care System, Saint Louis, MO, USA

Cite this as: $B M J 2022 ; 376: 0415$ http://dx.doi.org/10.1136/bmi.0415 Published: 17 February 2022

\title{
Mental health in people with covid-19
}

\section{Ziyad Al-Aly director}

In April 2021, we published a large-scale systematic characterisation of the clinical manifestations of long covid-the post-acute sequelae that result from an infection with SARS-CoV-2. ${ }^{1}$ We followed people for six months and showed that covid-19 can lead to post-acute sequelae in nearly every organ system, including increased risk of mental health disorders.

The research prompted calls from patients and healthcare practitioners to do a detailed analysis with longer follow-up to better understand the risks of the mental health outcomes experienced by people with covid-19.

In our recent study, published in The BMJ, we perform a comprehensive in-depth evaluation of the risks of mental health disorders in people with covid-19 at one year. ${ }^{2}$ The results show that even when compared to contemporaneous controls of people who did not have covid-19, but were exposed to the same adverse forces of the pandemic-including economic, social, and other stressors-those with covid-19 exhibited increased risk of mental health outcomes. This was consistent in analyses versus a historical control group from a pre-pandemic era.

Our research catalogues the breadth of post-acute mental health outcomes experienced by people with covid-19 including depression, anxiety, and stress and adjustment disorders. There was also evidence of increased risk of substance use disorders, neurocognitive decline, and sleep problems. These risks were evident even among those people whose disease was mild and did not require hospital admission for covid-19.

Altogether, the findings suggest that people with covid-19 are experiencing increased rates of mental health outcomes, which could have far-reaching consequences. The increased risk of opioid use is of particular concern, especially considering the high rates of opioid use disorders pre-pandemic. The increased risks of mental health outcomes in people with covid-19 demands greater attention now to mitigate much more serious downstream consequences in the future.

Some may use our findings to gaslight or dismiss long covid as a psychosomatic condition or explain the myriad manifestations of long covid as the result of mental illness. This dismissal is contrary to scientific evidence and is harmful to patients and communities. Mental health disorders represent one part of the multifaceted nature of long covid which can affect nearly every organ system (including the brain, heart, and kidneys). Our results should be used to promote awareness of this risk among people with covid-19 and to guide efforts for the early identification and treatment of affected individuals.

The body of evidence on long covid-from our work and others-suggests the need to reframe our thinking about SARS-CoV-2. It is not only a respiratory virus; it is a systemic virus that may provoke damage and clinical consequences in nearly every organ system-including mental health disorders and neurocognitive decline.

Long covid demands greater attention now to better understand it, prevent it, and treat affected individuals. International bodies, governments, and other stakeholders must pay attention. Failure to attend to the risks of long covid risks amplifying the profound losses we have experienced in this global pandemic.

Competing interests: Please see full declaration on linked research paper.

Provenance and peer review: Commissioned, not peer reviewed.

Al-Aly Z, Xie Y, Bowe B. High-dimensional characterization of post-acute sequelae of COVID-19. Nature 2021:594:259-64. doi: 10.1038/s41586-021-03553-9. pmid: 33887749

2 Xie Y, Xu E, Al-Aly Z. Risks of mental health outcomes in people with covid-19: cohort study. BMJ2022;376:e068993. doi: 10.1136/bmj-2021-068993. 